\title{
Special issue on the role of extracellular vesicles in human diseases
}

\author{
Yong Song Gho (1) and Jaewook Lee
}

Almost all cells on earth release vesicles, which are nano-sized enclosed structures with a lipid bilayer, into the extracellular environment ${ }^{1-4}$. Many different terms, such as exosomes and microvesicles, have been used for such vesicles, which can collectively be called extracellular vesicles ${ }^{5}$. Extracellular vesicles contain diverse bioactive components, such as proteins, lipids, nucleic acids, and metabolites ${ }^{6,7}$, that represent a subset of cellular components, implying the existence of cargo-specific sorting mechanisms ${ }^{8}$. These vesicles are present in biological fluids and environments and have been proposed to play diverse pathophysiological roles, such as immune modulation, angiogenesis, tissue repair, and tumor metastasis $^{9,10}$. Extracellular vesicle-mediated intercellular and interkingdom communication are evolutionarily conserved phenomena ${ }^{1-4}$. The field of extracellular vesicle research is intensively expanding, as indicated by a rapid increase in the number of extracellular vesicle-related publications collected in EVpedia (http://evpedia.info), a community web portal for extracellular vesicle research $^{11,12}$. Current research has suggested using extracellular vesicles as next-generation targets for diagnostics and therapeutics ${ }^{13,14}$. However, systematic and comprehensive studies based on emergent properties of these vesicles rather than reductionist approaches are essential for understanding the complexity of extracellular vesicle-mediated intercellular and interkingdom communication $^{1-4}$. The aim of this Special Issue of Experimental $\&$ Molecular Medicine, which is entitled "Role of Extracellular Vesicles in Human Diseases", is to highlight the significance of extracellular vesicles in human diseases.

In this Special Issue, we present five review articles that provide insights into current knowledge regarding the roles of extracellular vesicles in human diseases. Kim et al.

\footnotetext{
Correspondence: Yong Song Gho (ysgho@postech.ac.kr)

'Department of Life Sciences, Pohang University of Science and Technology (POSTECH), Pohang 37673, Republic of Korea
}

introduce proteome profiling of extracellular vesicles from different cancers using mass spectrometry-based proteomic approaches ${ }^{15}$. They specifically note the use of extracellular vesicle-associated proteins as predictive cancer biomarkers and the importance of enriched proteins in cancer-associated extracellular vesicles in cancer development and progression; thus, these vesicle-enriched proteins could be potential therapeutic targets. Buzás et al. present an overview of systems biology approaches to investigate the roles of extracellular vesicles in human diseases ${ }^{16}$. They provide examples of how such approaches can be used to identify correlations between genes involved in extracellular vesicle biogenesis and diseases. Ochiya and Kosaka et al. introduce current studies regarding extracellular vesicles as biomarkers for cancer diagnosis based on the biology of extracellular vesicles in cancer development ${ }^{17}$. They also introduce several technologies to detect extracellular vesicles to enable the use of extracellular vesicle-associated molecules for cancer diagnosis. Camussi et al. discuss the roles of extracellular vesicles in onco-nephrology ${ }^{18}$. They especially emphasize the role of renal cancer stem cell-derived extracellular vesicles in favoring tumor aggressiveness, angiogenesis, and immune modulation. Schiffelers and Vader et al. review current knowledge regarding the targeting, internalization, and intracellular trafficking of extracellular vesicles $^{19}$. They also provide engineering approaches that impact the circulation kinetics, bio-distribution, targeting, and internalization of extracellular vesicles.

We hope the review articles presented in this Special Issue of Experimental \& Molecular Medicine shed light on the significance of extracellular vesicles in human diseases. Steady research on the components and functions of extracellular vesicles would facilitate the use of extracellular vesicles in diagnostics and therapeutics. Finally, we appreciate the efforts of all contributors to this Special Issue. 


\section{Acknowledgements}

This work was supported by the National Research Foundation of Korea (NRF) grant funded by the Korea government (MSIT; grant no. 2018R1A2A1A05079510 to Y.S.G.).

\section{Conflict of interest}

The authors declare that they have no conflict of interest.

\section{Publisher's note}

Springer Nature remains neutral with regard to jurisdictional claims in published maps and institutional affiliations.

Received: 13 December 2018 Accepted: 14 December 2018

Published online: 15 March 2019

\section{References}

1. Gho, Y. S. \& Lee, C. Emergent properties of extracellular vesicles: a holistic approach to decode the complexity of intercellular communication networks. Mol. Biosyst. 13, 1291-1296 (2017).

2. Lee, E. Y. et al. Gram-positive bacteria produce membrane vesicles: proteomics-based characterization of Staphylococcus aureus-derived membrane vesicles. Proteomics 9, 5425-5436 (2009).

3. Kim, D. K. et al. EVpedia: a community web resource for prokaryotic and eukaryotic extracellular vesicles research. Semin. Cell Dev. Biol. 40, 4-7 (2015).

4. Kim, J. H., Lee, J., Park, J. \& Gho, Y. S. Gram-negative and Gram-positive bacterial extracellular vesicles. Semin. Cell Dev. Biol. 40, 97-104 (2015).

5. Choi, D. S., Kim, D. K, Kim, Y. K. \& Gho, Y. S. Proteomics of extracellular vesicles: Exosomes and ectosomes. Mass Spectrom. Rev. 34, 474-490 (2015).

6. Choi, D. S., Kim, D. K., Kim, Y. K. \& Gho, Y. S. Proteomics, transcriptomics and lipidomics of exosomes and ectosomes. Proteomics 13, 1554-1571 (2013).
7. Mayr, M. et al. Proteomics, metabolomics, and immunomics on microparticles derived from human atherosclerotic plaques. Circ. Cardiovasc. Genet. 2, 379-388 (2009).

8. Choi, D. S. et al. The protein interaction network of extracellular vesicles derived from human colorectal cancer cells. J. Proteome Res. 11, 1144-1151 (2012).

9. Kim, C. W. et al. Extracellular membrane vesicles from tumor cells promote angiogenesis via sphingomyelin. Cancer Res. 62, 6312-6317 (2002).

10. Yoon, Y. J., Kim, O. Y. \& Gho, Y. S. Extracellular vesicles as emerging intercellular communicasomes. BMB Rep. 47, 531-539 (2014).

11. Kim, D. K. et al. EVpedia: an integrated database of high-throughput data for systemic analyses of extracellular vesicles. J. Extracell. Vesicles 2, 20384 (2013).

12. Kim, D. K. et al. EVpedia: a community web portal for extracellular vesicles research. Bioinformatics 31, 933-939 (2015).

13. Choi, D. S., Lee, J., Go, G., Kim, Y. K. \& Gho, Y. S. Circulating extracellular vesicles in cancer diagnosis and monitoring: an appraisal of clinical potential. Mol. Diagn. Ther. 17, 265-271 (2013).

14. Gyorgy, B., Hung, M. E., Breakefield, X. O. \& Leonard, J. N. Therapeutic applications of extracellular vesicles: clinical promise and open questions. Annu. Rev. Pharmacol. Toxicol. 55, 439-464 (2015).

15. Bandu, R., Oh, J. W., \& Kim, K. P. Mass spectrometry-based proteomic profiling of extracellular vesicles and their roles in cancer biology. Exp. Mol. Med. (2018). https://doi.org/10.1038/s12276-019-0218-2.

16. Gézsi, A., Kovács, Á., Visnovitz, T., \& Buzás, E. I. Systems biology approaches to investigate the roles of extracellular vesicles in human diseases. Exp. Mol. Med. (2018). https://doi.org/10.1038/s12276-019-0226-2

17. Kosaka, N., et al. Exploiting the message from cancer: diagnostic value of extracellular vesicles for clinical application. Exp. Mol. Med. (2018). https:/doi. org/10.1038/s12276-019-0219-1

18. Gai, C., Pomatto, M. A. C., Grange, C., Deregibus, M. C., \& Camussi, G. Extracellular vesicles in onco-nephrology. Exp. Mol. Med. (2018). https://doi.org/ 10.1038/s12276-019-0213-7

19. Murphy, D. E., et al. Extracellular vesicle-based therapeutics: natural versus engineered targeting and trafficking. Exp. Mol. Med. (2018). https://doi.org/ 10.1038/s12276-019-0223-5 